\title{
Size effects in finite systems with long-range interactions
}

\author{
E. S. Loscar \\ Instituto de Física de Líquidos y Sistemas Biológicos (IFLYSIB), UNLP, CCT La Plata-CONICET, \\ Calle 59 no. 789, B1900BTE La Plata, Argentina \\ and Departamento de Física, Universidad Nacional de La Plata, c.c. 67, 1900 La Plata, Argentina \\ C. M. Horowitz \\ Instituto de Investigaciones Fisicoquímicas Teóricas y Aplicadas (INIFTA), UNLP, CCT La Plata-CONICET, \\ Sucursal 4, Casilla de Correo 16, (1900) La Plata, Argentina
}

(Received 7 June 2017; revised manuscript received 23 December 2017; published 6 March 2018)

\begin{abstract}
Small systems consisting of particles interacting with long-range potentials exhibit enormous size effects. The Tsallis conjecture [Tsallis, Fractals 3, 541 (1995)], valid for translationally invariant systems with long-range interactions, states a well-known scaling relating different sizes. Here we propose to generalize this conjecture to systems with this symmetry broken, by adjusting one parameter that determines an effective distance to compute the strength of the interaction. We apply this proposal to the one-dimensional Ising model with ferromagnetic interactions that decay as $1 / r^{1+\sigma}$ in the region where the model has a finite critical temperature. We demonstrate the convenience of using this generalization to study finite-size effects, and we compare this approach with the finite-size scaling theory.
\end{abstract}

DOI: 10.1103/PhysRevE.97.032103

\section{INTRODUCTION}

Physical systems with a finite number of constituent particles show a size-dependent behavior. Nowadays experimental systems on the order of nanometers, involving a few atoms, are intensively and widely investigated. These objects, called ultrafine particles or nanoparticles, present the strong dependence of their physical behavior on their size as a main signature. This property makes them of great importance to the scientific community due to current and potential applications. An example is the case of nanosized magnetic particles that have applications in high-density recording, color imaging, ferrofluids, high-frequency devices, and magnetic refrigerators $[1,2]$. In this kind of system the magnetization as a function of the temperature at null magnetic field, the effective Curie temperature, and its dispersion have a clearly different behavior for different sizes [3-8].

Also, in nanosystems where long-range (LR) interactions are present, enormous finite-size effects (FSEs) are expected. The understanding of this type of interactions in nanosystems is of great importance due to its possible application in diverse research areas and to the predominant role of these interactions in the synthesis, design, and manipulation of nanomaterials [9]. In spite of the simple explanation of these enormous FSEs, i.e., that for a finite system the long-range interactions are delimited by the size of the system, leading to a strongly sizedependent behavior, there is no general theoretical framework properly describing this kind of experiment or simulation. In this context, two general questions arise naturally: (1) How should the results for two systems of different size be related? (2) What is the relationship of these results with the bulk behavior?

From a classical thermodynamic point of view, the bulk properties are free of any edge effect. Strategies for obtaining this bulk behavior are based on minimizing the FSEs by increasing the size of the sample and/or applying periodic boundary conditions (PBCs). So in this context the FSEs are undesirable and should be minimized. In the study of nanosystems with LR interactions it is obvious that the strategy to follow must be different. A priori, the following two well-founded approaches are available for studying finite-size systems.

In the renormalization group (RG) theory of critical phenomena, the characteristic spatial length of the system can be introduced as a new relevant parameter, and so, based on this theory, the finite-size effects can be used to determine both the critical parameters and critical exponents [10-12]. The general $\mathrm{RG}$ ideas have been applied in the context of confined systems with LR interactions in several works [13-18]. With this theory, the knowledge of the scaling function, the critical exponents, and critical parameters allows understanding and predicting the behavior of a finite system but only in the proximity of the critical temperature. However, when the system size is too small, the number of degrees of freedom may not be enough to apply properly the standard statistical mechanics and the use of the theory would not be well founded. Therefore, there is a minimal size from which the theory can be applied to describe a finite system. In spite of this fact, in the literature, the finite-size critical theory has been employed in different systems with long-range interactions in the context of nanoparticle studies $[7,8,19,20]$.

Another kind of finite-size scaling for systems with longrange interactions can be regarded using the so-called Tsallis conjecture. Basically this conjecture has been designed for spatially invariant systems (where all sites are equivalent) and relates different system sizes by means of a rescaling of the parameters (field and temperature) with the interaction strength [21,22]. Despite being tested in LR systems, this theoretical conjecture has been little exploited to describe finite-size 
effects [22,23]. Nanosystems with LR interactions cannot fulfill the condition of invariance required for the validity of this approach. Taking this into account, the aim of this work is to generalize this theory to describe this kind of system. In order to do that, we will use a minimalist one-dimensional magnetic model with finite critical temperature and open boundary conditions (OBCs), in which we can control the decay of LR interactions.

The paper is organized as follows: in Sec. II the model and computational details are presented; in Sec. III two possible approaches to study the finite-size effects are detailed. The results are reported in Section IV, and finally, Section V is devoted to our conclusions.

\section{MODEL AND COMPUTATIONAL DETAILS}

We consider the one-dimensional Ising model with longrange interactions given by the Hamiltonian

$$
\mathcal{H}=-J \sum_{i, j=1}^{N} \frac{s_{i} s_{j}}{|j-i|^{1+\sigma}},
$$

where $J$ is the coupling constant $(J>0), 1+\sigma$ is an exponent that controls the decay of LR interactions $(\sigma>-1), s_{i}= \pm 1$ is the classical Ising spin at site $i, N$ is the number of spins in the chain, and $|j-i|$ is the distance between spins $i$ and $j$. The summation in this Hamiltonian is extended to all pairs of spins.

For the case of $\sigma \leqslant 0$, in the asymptotic regime $N \rightarrow \infty$, the energy is divergent or nonextensive, while in the same asymptotic regime, the system is extensive for $\sigma>0$ and presents different behaviors. Ruelle [24] rigorously proved the absence of a long-range order for $\sigma>1$, while Dyson [25] demonstrated the existence of a phase transition between ferromagnetic and paramagnetic phases in the range $0<\sigma<1$. Using different methods [26-31], it has been established that the critical exponents are classical for $0<\sigma \leqslant 0.5$ (mean-field behavior) and continuously varying values for $0.5<\sigma \leqslant 1$ (long-range behavior). For $\sigma>1$ the system does not exhibit phase transition, like the usual one-dimensional Ising model (short-range behavior).

In order to mimic very small systems, including sizes compatible with nanosystems, we performed our simulations using samples with $100 \leq N \leq 5000$ and taking OBCs. The spin update was performed by means of the single spin-flip Monte Carlo dynamics (Metropolis) where a Monte Carlo time step (MCS) is defined when all the spins of the sample are updated once on average. We set the Boltzmann constant equal to unity throughout; temperature and energy were measured in units of $J$.

During the simulations, we recorded the time dependence of the total magnetization and its susceptibility, which are, respectively, given by

$$
M(N, T)=\sum_{i=1}^{N}\left\langle s_{i}\right\rangle
$$

and

$$
\chi_{T}=\frac{1}{N}\left(\left\langle M^{2}\right\rangle-\langle|M|\rangle^{2}\right),
$$

where $\langle\cdots\rangle$ indicates time averages.

\section{TREATMENTS OF THE FINITE SIZE}

In this section, we present two possible approaches to study finite-size effects: on the one hand, the standard scaling coming from the critical theory and, on the other, our proposed generalization of the Tsallis conjecture.

\section{A. Finite-size scaling theory}

The onset of the critical behavior is associated with the nonanalyticity of the free energy. This produces scale invariance and divergent susceptibilities in the form of power laws at the critical point. However, nonanalyticities are not possible in finite chains. So, near the critical temperature, finite-size scaling appears as power laws in terms of the linear dimensions $L$ of the systems (in our model $L=N$ ).

The standard scaling that describes the form that the order parameter (or magnetization per site) $m(T)$ goes to zero at the critical temperature $T_{c}$ is

$$
m\left(T_{c}\right)=m_{o} L^{-\beta / v}
$$

where $\beta$ and $\nu$ are the order parameter and length correlation critical exponents, respectively. The shift in the so-called pseudocritical temperature $T_{c}(L)$ (taken as the peak of the susceptibility $\chi_{T}$ ) is given by

$$
T_{c}-T_{c}(L) \propto L^{-1 / v},
$$

while the maximum of the magnetic susceptibility behaves as the power law

$$
\chi_{\max } \propto L^{\gamma / v}
$$

where $\gamma$ is the critical exponent associated with the magnetic susceptibility with null field.

Using Eqs. (4)-(6) the data corresponding to magnetization and susceptibility for different systems sizes can be collapsed in a region near the critical point. More specifically, the scaled magnetization $|m| L^{\beta / v}$ versus $\psi=\left(1-T / T_{c}\right) L^{1 / v}$ and $\chi_{T} / L^{\gamma / v}$ versus $\psi$ should not depend on the system size in that region.

The finite-size laws described before are valid using PBCs and OBCs for short-range interactions [32]. In the following the finite-size laws which we will assume for the one-dimensional Ising model with LR interactions are detailed.

\section{B. Finite-size scaling theory in the mean-field region}

Above the upper critical dimension the finite-size laws have been recently studied in Refs. [33-35]. This regime, in the case of the one-dimensional Ising model with long-range interactions, is given by the condition $\sigma<1 / 2$. The critical exponents for the LR interactions given by Eq. (1) in this region are the mean field exponents except that $\nu=1 / \sigma$ and the correlation critical exponent $\eta=2-\sigma$ [36]. However, the previous standard scalings [Eqs. (4)-6)] have to be modified due to the occurrence of a dangerous irrelevant variable [33]. These scalings must be transformed according at which temperature (critical or pseudocritical) the physical quantities are evaluated and depending on the boundary conditions [34]. Incorporating the dangerous irrelevant variable, $\mathrm{RG}$ prediction determined the critical behavior called QFSS scaling [33]. On the one hand, the susceptibility evaluated at the pseudocritical 
temperature and using OBCs is affected by this variable, then QFSS holds:

$$
\chi_{\max } \propto L^{\theta}, \quad \theta=1 / 2 .
$$

On the other hand, this variable does not affect the behaviors of the magnetization evaluated at $T_{c}$ and the critical shift. Then, in these cases, the critical behavior is controlled by the Gaussian fixed point [34]:

$$
\begin{gathered}
m\left(T_{c}\right)=m_{o} L^{-\frac{1-\sigma}{2}}, \\
T_{c}-T_{c}(L) \propto L^{-\lambda}, \quad \lambda=1 / v=\sigma .
\end{gathered}
$$

Also, at the critical dimension given by the limit value of the interaction $\sigma=1 / 2$, logarithmic corrections must be used in the case of scaling affected by the dangerous irrelevant variable [35]. Then, instead of Eq. (7), we have

$$
\chi_{\max } \propto L^{\theta} \sqrt{\ln (L)}, \quad \theta=1 / 2 .
$$

\section{Finite-size scaling theory in the long-range region}

Below the upper critical dimension $(1 / 2<\sigma$ in our case) the finite-size laws with long-range interaction are less explored. In particular, to the best of our knowledge, the validity of the power laws given by Eqs. (4)-(6) has not been studied with the OBCs used in the present work. These power laws could be a oversimplification of the finite-size effects because this kind of boundaries can give rise to relevant scaling variables $[14,15]$. However, several works suggest that the finite-size scaling ansatz is reasonable for the case of the onedimensional Ising model with LR interactions. In this model, Glumac and Uzelac have introduced the so-called finite range scaling [37]. They consider the infinite-length model by means of the transfer matrix method. In this treatment, the interactions are truncated to neighbors at distance $r>R_{\text {cut }}$, where $R_{\text {cut }}$ indicates the range or cutoff of the interactions. Invoking the scaling hypothesis on the cutoff $R_{\text {cut }}$, they obtain analogous equations to the standard finite-size scaling with the cutoff $R_{\text {cut }}$ playing the role of the length $L$ (i.e., $R_{\text {cut }} \rightarrow L$ ). Their results [38] show critical exponents similar to those posteriorly obtained by other methods in Refs. [27,31,39]. Studying the same model for $\sigma=0.75$ and PBCs, Rodriguez et al. [27] have shown that the pseudocritical temperature, calculated by the short-time dynamics technique, depend on the cutoff $R_{\text {cut }}$, and it is independent of the length $L$. These authors, based on the results of Ref. [38] extrapolated the critical temperature by means of Eq. (5) making $R_{\text {cut }} \rightarrow L$. Therefore, from these works, one can conclude that with long-range interactions, the cutoff $R_{\text {cut }}$ governs the scaling behavior, which is analogous to the standard finite-size scaling. Then, in the case of systems in which PBCs are applied making the cutoff $R_{\text {cut }}=L / 2$, the finite range scaling produces power laws dependent on the length $L$. For example in Refs. [40,41] Eqs. (4)-(6) have been verified in a one- and in a two-dimensional continuous Ising model by applying PBCs in this way.

Returning to the case of OBC conditions used in this work, the cutoff is imposed by the confinement of the system, in such way that it results inhomogeneous. However, one can still assume the existence of an effective cutoff that scales with $L$. So, considering this hypothesis and the previous mentioned works, we will assume the ansatz of finite-size scaling giving by Eqs. (4)-(6).

\section{Tsallis conjecture and its generalization}

From a thermodynamical point of view, one expects the Hamiltonian given by Eq. (1) to produce extensive energy $U$ and magnetization $M$, respectively:

$$
U \propto N \widetilde{u}(T, \sigma)
$$

and

$$
M \propto N \tilde{m}(T, \sigma),
$$

where $\widetilde{u}(T, \sigma)$ and $\widetilde{m}(T, \sigma)$ are functions only of the temperature and $\sigma$. However, when the interaction is too strong, these observables are not linear functions of $N$. Then for finite $N$ this unexpected behavior implies large and nonstandard (i.e., noncritical) FSEs that are present in all the range $-1<\sigma<1$.

The Tsallis conjecture is stated for general systems with LR interactions. In our case it states that the energy and magnetization of a finite system of $N$ constituents can be expressed as follows, rather than by Eqs. (11) and (12):

$$
\begin{gathered}
U(N, T) / N^{*} \propto N u^{\prime}\left(T^{*}\right), \\
M(N, T) \propto N m^{\prime}\left(T^{*}\right)
\end{gathered}
$$

where $T^{*}$ is given by

$$
T^{*}=T / N^{*} .
$$

The key quantity $N^{*}(N, \sigma)$ is related to the $T=0$ internal energy per particle and characterizes the effective number of neighbors that can be associated with a given particle in a translationally invariant system, i.e., a system where all particles are equivalent. This quantity has different versions in the literature, but in all cases (with $\sigma \neq 0$ ), it can be expressed as

$$
N^{*}(N, \sigma)=\mathrm{C} \int_{1}^{R} \frac{1}{r^{1+\sigma}} d r=\frac{\mathrm{C}}{\sigma}\left[1-R^{-\sigma}\right],
$$

where $\mathrm{C}$ is a constant and $R=\frac{N}{a}$ ( $a=1$ in Ref. [30], $a=(1+\sigma)^{\frac{-1}{\sigma}}$ in Ref. [42], and $a=2$ in Refs. [22,43]). In Ref. [30] it has been shown that the quantity $N^{*}(N, \sigma)$ makes the divergent nonextensive case of $\sigma<0$ treatable.

Here we are interested in the extensive case of $0<\sigma<1$, in which there is a finite critical temperature $T_{c}$. In this case, the asymptotic regime $N \rightarrow \infty$ corresponds to the well-defined bulk behavior. Taking this asymptotic regime in Eq. (16), from Eq. (15) we obtain $T^{*} \rightarrow \sigma T / C$. Therefore, in order to have a variable $T^{*}$ that allows us to compare the results from finite system with the bulk behavior, we set $\mathrm{C}=\sigma$. With these conditions the Tsallis conjecture, given by Eqs. (13)-(15), can be interpreted as follows. A system of size $N^{\prime}$ at temperature $T^{\prime}$ with a scaled temperature $T_{0}^{*}=T^{\prime} / N^{*}\left(N^{\prime}, \sigma\right)$ should have a similar behavior to that of another system of size $N^{\prime \prime}$ at temperature $T^{\prime \prime}$, provided that the latter is at the same scaled temperature, i.e., $T_{0}^{*}=T^{\prime \prime} / N^{*}\left(N^{\prime \prime}, \sigma\right)$. This argument can also be applied to the infinite system with $N \rightarrow \infty$, and a more relevant consequence emerges; that is, the two systems should have a similar behavior to that of the infinite system (i.e., the bulk behavior) at temperature $T=T_{0}^{*}$. In this way, in principle, 
we can interpret $T^{*}$ as the temperature at which the bulk has properties similar to that of our finite system with scaled temperature $T^{*}$.

We remark that Eqs. (13)-(15) are valid for translationally invariant systems. In the present work we are interested in systems with open boundary conditions, which break down this requirement. However, we can consider this difference by the following proposal. We interpret Eq. (16) as the strength of the interaction for a system with equivalent particles that are separated until a maximum distance $R\left(R=R_{\text {cut }}\right.$, the cutoff in previous section). For example, $R=N / 2$ in the case of a one-dimensional system of size $N$ with PBCs. In our case, due to the existence of edges, the Ising chain with OBCs should be more weakly connected than a system with PBCs (ring configuration). This decrement in connectivity can be thought as a reduction of the strength of interactions. So, due to the border effects, for the long-range behavior $(0<\sigma<1)$ we propose using the same Eq. (16) but with an effective maximum distance $R_{0}$. In order to do that we can adjust, in a controlled way, the constant $a$ (which will be called $a_{0}$ ) resulting in a maximum empirical distance $R_{0}=N / a_{0}$. It should be noted that it is equivalent to assuming the most simple scaling for the maximum distance as $R_{0} \propto N$. Therefore, with this interpretation, we expect a shorter maximum distance $R_{0}$ for the case of OBCs than for PBCs; that is, numerically we expect $a_{0} \gtrsim 2$.

In conclusion, we will assume the Tsallis conjecture with the rescaling for the temperature given by

$$
T^{*}=\frac{T}{1-\left[\frac{a_{0}}{N}\right]^{\sigma}},
$$

where, in order to consider the interaction cutoff due to OBCs, $a_{0}$ is an adjustable parameter. We will adjust $a_{0}$ in such way that our results for the finite system collapse using the variable $T^{*}$, which is the essence of the Tsallis conjecture.

To clarify the previous discussion, in Fig. 1 we plot the results from a simulation using OBCs. In Fig. 1(a) we can see the temporal evolution of the absolute magnetization starting in the ordered state $\left(m_{0}=1\right)$ at temperature $T=5$ for the case of $\sigma=0.3$. The data for the smaller sizes $N=1000,5000$ are the average of $n_{s}=100$ and 10 independent runs, respectively. This figure shows, for the indicated lattice sizes, enormous finite-size effects. In contrast, Fig. 1(b) shows that the results for the same kind of simulation done for $T^{*}=5$ practically coincide. In this latter case, for each size, we inverted Eq. (17) and used the value $a_{0}=3.3$ as obtained in the next section by means of a collapse (for $T^{*}=5$ the temperatures are $T \approx 4.10,4.44$, and 4.63 for $N=1000,5000,20000$, respectively).

It is worth noting that, in contrast with the finite-size scaling described in Sec. III A, our proposed Tsallis scaling is independent of the critical exponents and the critical temperature $T_{c}$. Basically, it depends on the exponent that controls the decay of LR interactions $(1+\sigma)$.

\section{RESULTS AND DISCUSSION}

We simulated the one-dimensional Ising model with the interaction power-law decay with OBCs using $\sigma=0.10,0.30,0.50,0.75$, and 0.90 . We obtained stationary
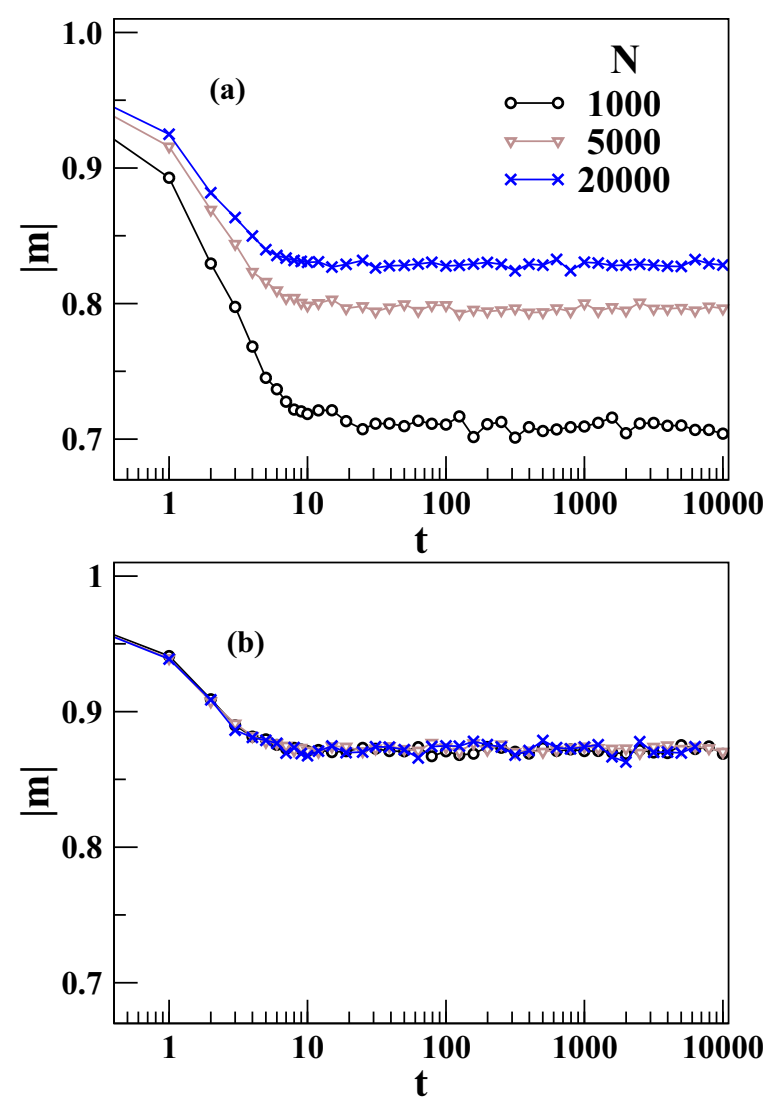

FIG. 1. (a) Evolution of the absolute magnetization starting from the ordered state $\left(m_{0}=1\right)$ for $\sigma=0.3$ and different lattice sizes at temperature $T=5$. (b) Same as (a) at $T^{*}=5$ using $a_{0}=3.3$ and Eq. (17).

values of absolute magnetization and susceptibility by means of the temporal evolution at different temperatures. Specifically, we start our simulation from the ferromagnetic phase (i.e., $s_{i}=1 \quad \forall i$ ) and let the system relax for $\tau_{\text {Relax }}=10^{4}-10^{5}$ MCSs. After that, we average the observables recorded until $\tau_{\text {Max }}=10^{6}$ MCSs.

We will present our results according to the different behaviors given by the value of $\sigma$, i.e., the mean-field region $(0<\sigma \leqslant 0.5)$ and long-range region $(0.5<\sigma<1)$.

\section{A. Mean-field region}

In this section we present the results obtained for $\sigma=$ $0.10,0.30$, and 0.50 . Figure 2 shows the results for the case of $\sigma=0.30$ for systems with OBCs and sizes $100 \leqslant N \leqslant 5000$. The absolute magnetization and the magnetic susceptibility, against the temperature $T$, are shown in Figs. 2(a) and 2(b), respectively. The critical temperature $T_{c} \approx 7.347$, taken from Ref. [31], is indicated by a vertical dashed line. As we can observe, both observables present enormous finite-size effects in all the range of temperature, even far from $T_{c}$.

In Fig. 2 the black squares joined by a solid line are the results from simulations that we did for the bulk behavior. These data were obtained using the infinite-image method [44]. This method has proved to be very convenient to reproduce the bulk properties in this model, since it minimizes size 

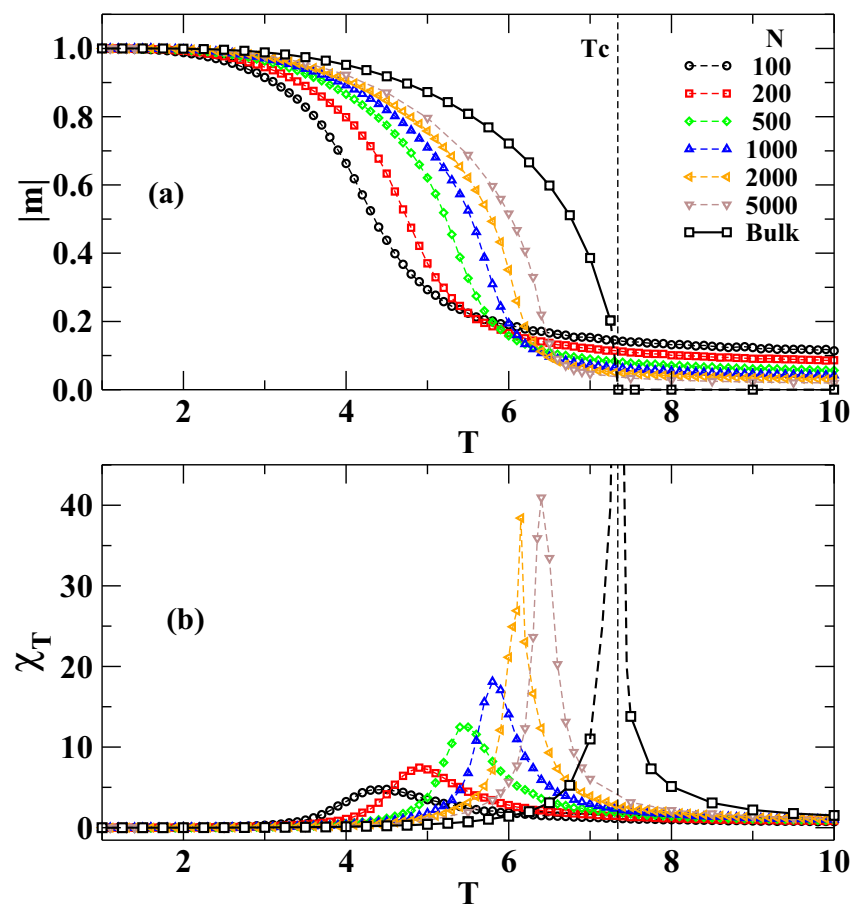

FIG. 2. (a) Absolute magnetization vs $T$ and (b) susceptibility vs $T$ for $\sigma=0.3$ and the indicated sizes $100 \leqslant N \leqslant 5000$. The black squares joined by the solid line are data obtained for the bulk, while dashed lines are a qualitative continuation of this curve. The vertical dashed lines represent the critical temperature of the system taken from Ref. [31].

effects and rapidly the results converge to the thermodynamics limit $[28,45]$. Basically, the infinite-image method uses periodic boundary conditions, which are implemented by tiling the entire space with infinite replicas of the original finite system. Due to the periodicity of the aggregation, i.e., the original system plus all of its replicas, the Hamiltonian of the aggregation can be reduced to a general Ising model Hamiltonian with effective interaction coefficients in the original finite system (for more details about the method see Refs. [44] and [46]). We checked that the result, obtained with this method, shown in Fig. 2 is size independent for $T<095 T_{c}$. Simulations with the original system size $N=2000$ and $N=5000$ have no differences, indicating that the bulk behavior has been reached at those temperatures.

We studied whether the finite-size data of Fig. 2 can be described using the finite-size scaling theory described in Sec. III A. Figure 3(a) shows log-log plots of $\left|m\left(T_{c}\right)\right|$ obtained from Fig. 2(a) as a function of the size $N$. As we can see, the data clearly suggest a power-law behavior. The best fit gives $\left|m\left(T_{c}\right)\right| \propto N^{-0.355}$, which is in agreement with the expected mean-field behavior given by Eq. (8). In Fig. 3(b) we plot the difference between the pseudocritical temperature $T_{c}(N)$, given by the peak of susceptibility $\chi_{T}$ from Fig. 2(b) for each size $N$, and the infinite-size critical temperature $T_{c}=7.347$ against the size $N$. As in the previous case, the data suggest a power-law behavior, and the best fit gives $T_{c}-T_{c}(N) \propto$ $N^{-0.29}$. Again, it is in agreement with the expected mean-field behavior given by Eq. (9). Finally, in Fig. 3(c), we plot the maximum value of the susceptibility $\chi_{\max }$ against $N$. Once
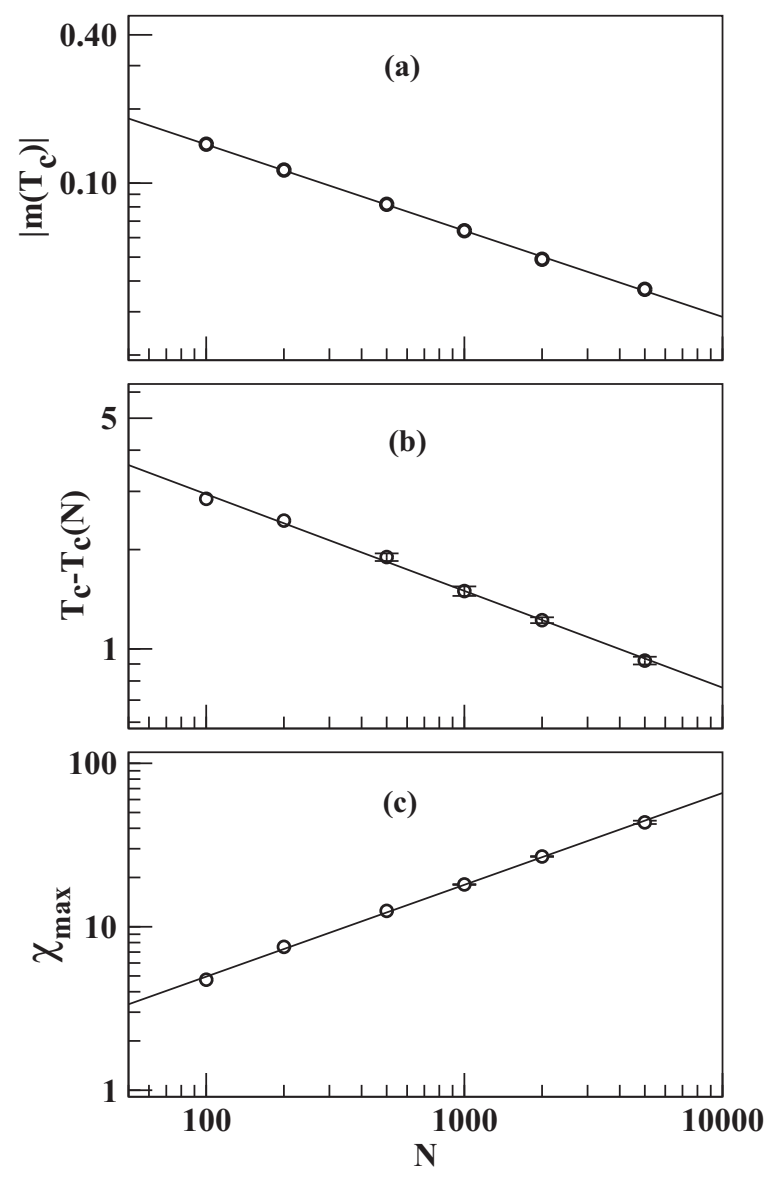

FIG. 3. Panels (a-c) The quantities corresponding to Eqs. (4)-(6), respectively, plotted against the system size $N$ in log-log plots. The fitted exponents are listed in Table I.

again, the data clearly suggest a power-law behavior, and the best fit gives $\chi_{\max } \propto N^{0.56}$, which differs slightly from the theoretical prediction given by Eq. (7).

We did the same analysis for $\sigma=0.10$ and $\sigma=0.50$ and found, as in the previous case, that the data can be fitted with power laws. Table I shows the values of the exponents obtained by fitting our data. In the case of $\sigma=0.50$, for the susceptibility we include the logarithmic correction given by Eq. (10). As we can see, these values are consistent with the mean-field exponents except for the case related to $T_{c}-T_{c}(N)$ for $\sigma=0.50$, where the exponent is significantly less than the expected theoretical value. We attribute this discrepancy to

TABLE I. Summary of the results obtained in the mean-field region using different range interactions. The theoretical exponents given by Eqs. (8), (9), and (7) are listed along with those obtained in this work, which are shown in the columns indicated by $\left(^{*}\right)$. In the second column we list the critical temperature used in the fits of Eqs. (8) and (9). The parameter $a_{0}$ adjusted to generalize the Tsallis conjecture is shown in the last column.

\begin{tabular}{lcccccccc}
\hline \hline$\sigma$ & $T_{c}$ & $\frac{1-\sigma}{2}$ & $\left(^{*}\right)$ & $1 / v$ & $\left(^{*}\right)$ & $\theta$ & $\left(^{*}\right)$ & $a_{0}$ \\
\hline 0.10 & 21.00 & 0.45 & $0.448(5)$ & 0.10 & $0.100(5)$ & 0.50 & $0.51(1)$ & 2.0 \\
0.30 & 7.347 & 0.35 & $0.355(5)$ & 0.30 & $0.29(1)$ & 0.50 & $0.56(2)$ & 3.3 \\
0.50 & 4.364 & 0.25 & $0.24(2)$ & 0.50 & $0.31(2)$ & 0.50 & $0.54(2)$ & 5.0 \\
\hline \hline
\end{tabular}



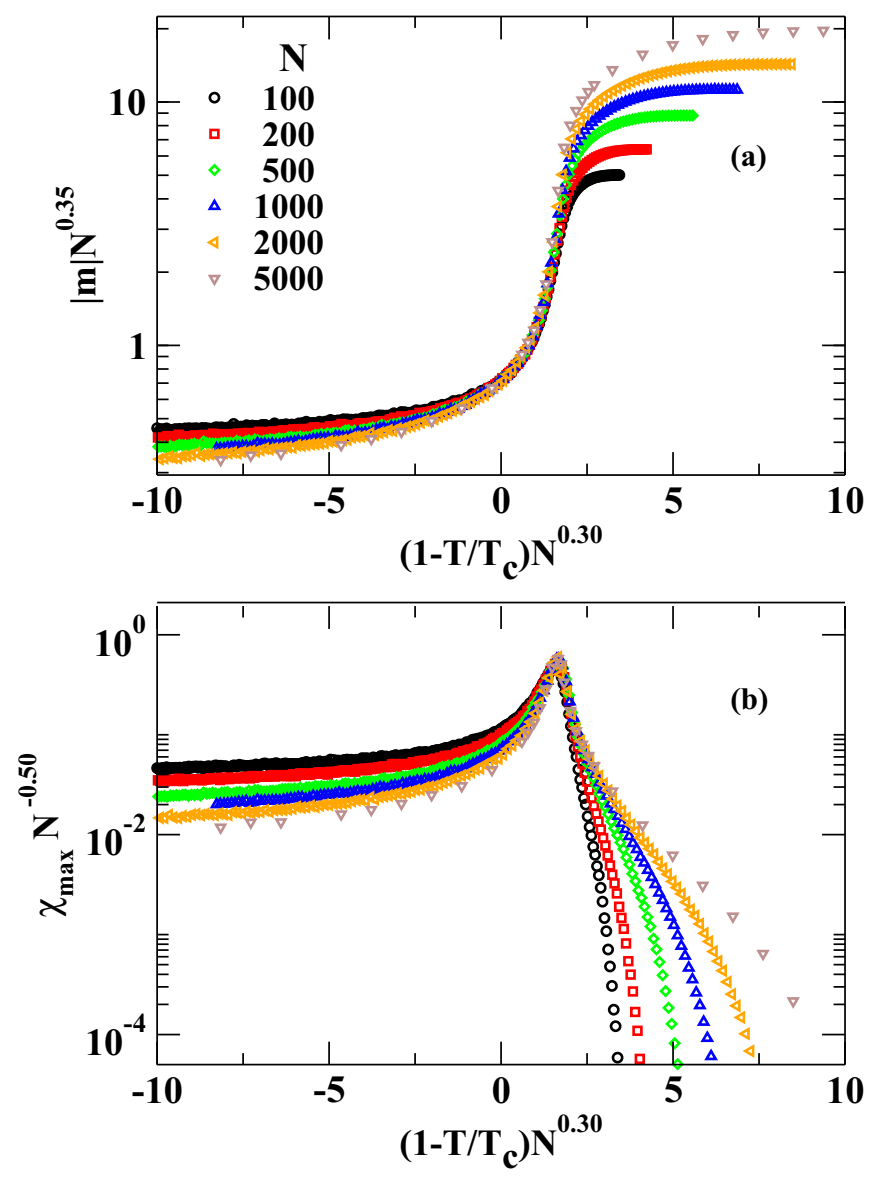

FIG. 4. (a) and (b) The collapses for magnetization and susceptibility, respectively, obtained from the data shown in Fig. 2 and using the theoretical exponents listed in Table I.

the small sizes of our simulated systems $(100 \leqslant N \leqslant 5000)$. Using larger systems, we expect that the value of this exponent approaches the mean-field value.

Figure 4(a) shows a standard collapse using $\psi=$ $\left(1-T / T_{c}\right) N^{1 / v}$ as abscissa and the scaled magnetization $|m| N^{(1-\sigma) / 2}$ as ordinate. This figure clearly shows that near $T_{c}$ there is a good collapse, but it fails for $|\psi| \gtrsim 1$. In Fig. 4(b) we plot the standard collapse for the susceptibility, i.e., $\chi_{T} N^{-1 / 2}$ versus $\psi$. In this case, the range of the collapse is restricted to a narrow interval given by $1.5 \lesssim \psi \lesssim 2.5$, which represents the interval $0.8 \lesssim T / T_{c} \lesssim 0.9$ for $N=5000$ and the interval $0.73 \lesssim T / T_{c} \lesssim 0.84$ for $N=2000$

From another point of view, we studied how well our generalization of the Tsallis conjecture works. In order to test this conjecture, we collapsed the data of the absolute magnetization presented in Fig. 2 with the data corresponding to the bulk. This was done using the magnetization obtained with OBCs and sizes $100 \leqslant N \leqslant 5000$. In order to obtain the best collapse we plotted these data against the rescaled temperature $T^{*}$ in the horizontal axis, and we adjusted the constant $a_{0}$ in Eq. (17). For the bulk data we used the identity $T^{*}=T$ in this axis. Figure 5(a) shows the best rescaling given by a value of $a_{0}=3.3$. The collapse works very well for temperatures lower than $T_{c}$ and also near $T_{c}$. Actually, for these sizes, this figure shows a good collapse until $T^{*} \lesssim 0.95 T_{c}$.
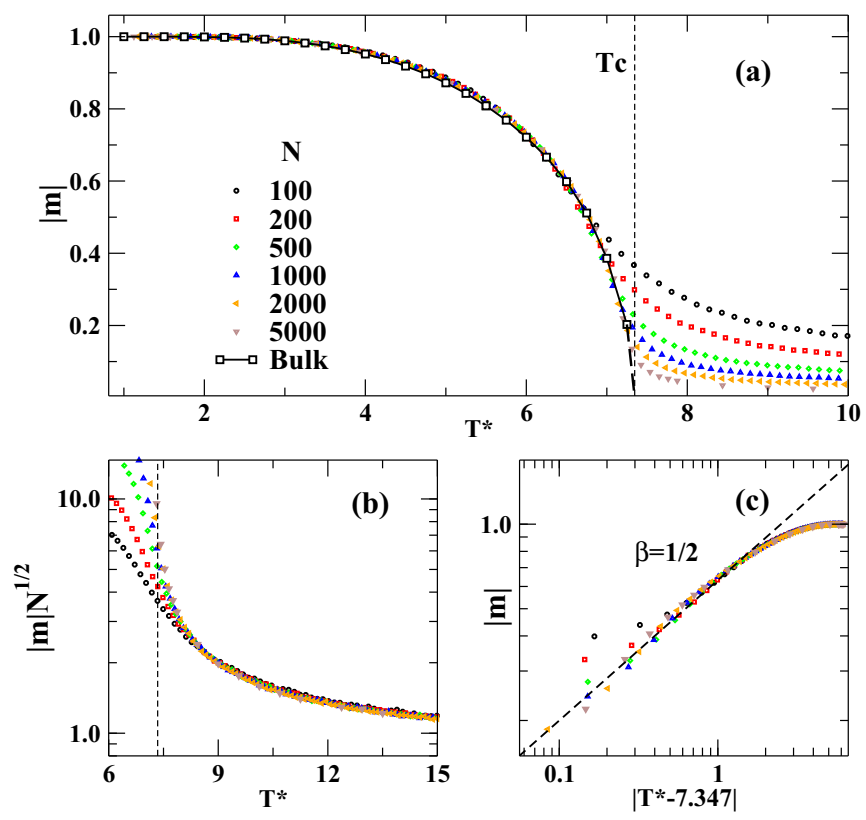

FIG. 5. Generalized Tsallis scaling for the magnetization using $T^{*}$ given by Eq. (17). (a) Absolute magnetization as a function of $T^{*}$ for different sizes as indicated. The black squares joined by the solid line are bulk results. (b) In the paramagnetic phase $|m| N^{1 / 2}$ is a function (size independent) of $T^{*}$ and corresponds to normal fluctuation. (c) Log-log plots of $|m|$ vs $\left|T^{*}-T_{c}\right|$. The dashed line is a power law given by Eq. (18) with exponent $\beta=1 / 2$.

Also, it is important to note that the best collapse between finite lattices (regardless of the bulk data) is also in agreement with the curve corresponding to the bulk.

Furthermore, Fig. 5(b) shows that, for values of the rescaled temperature $T^{*}$ greater than $T_{c}$, the behavior of the absolute magnetization is $|m|=O\left(N^{-1 / 2}\right)$. If one considers, for these temperatures, that the magnetization has a distribution centered at $m=0$, the quantity $\langle|m|\rangle$ represents the width of this distribution. Then, as the magnetization is a thermodynamic variable, its fluctuations $\sigma_{m}$ must behave as $\sigma_{m}=O\left(N^{-1 / 2}\right)$ and therefore $|m| \sim \sigma_{m}=O\left(N^{-1 / 2}\right)$. We remark that this simple standard scaling is not valid for the temperature $T$, but it appears when the temperature is rescaled with the generalized Tsallis scaling. In this way, $T^{*}$ seems to be a convenient variable to study the thermodynamic behavior.

Also, the critical behavior of the order parameter given by the power law

$$
|m| \sim\left|T-T_{c}\right|^{\beta},
$$

where $T_{c}$ is the critical temperature, is observed using the rescaled temperature $T^{*}$. Figure 5(c) shows the order parameter against $\left|T^{*}-T_{c}\right|$. The dashed line is a power law with the theoretical exponent $\beta=1 / 2$. In this way, $T^{*}$ shows an acceptable validity of Eq. (18). In contrast, using the temperature $T$ the power law is completely unobservable. Again, this is a confirmation that our rescaled results converge to the thermodynamic limit, and therefore the rescaled temperature $T^{*}$ has the basic thermodynamic critical behavior.

According to the Tsallis conjecture, other thermodynamic observables also should be a size-independent function of 


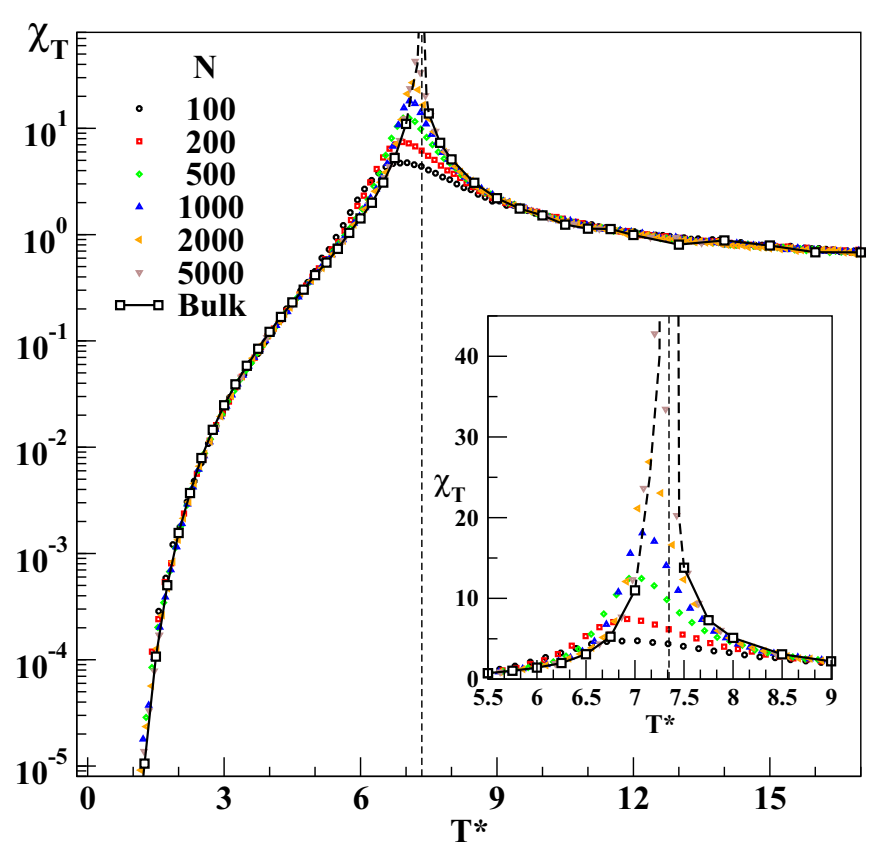

FIG. 6. Susceptibility $\chi_{T}$ as a function of the rescaled temperature $T^{*}$ for different sizes as indicated. The black squares joined by the solid line are bulk results as a function of $T$. The inset is a zoom of the critical region.

$T^{*}$ [21,22]. Figure 6 shows the magnetic susceptibility $\chi_{T}$ as a function of $T^{*}$ (using $a_{0}=3.3$ calculated above) and the results corresponding to the bulk. Again, we find a good agreement between the results of different sizes and the bulk. We can observe a good collapse from $10^{-5}$ to $10^{1}$, over six decades of the values of $\chi_{T}$. Note from Fig. 2(b) that the same data plotted against $T$ are very different and far from the bulk. As is shown in the inset of Fig. 6, in a small region around $T_{c}$ finite-size data plotted against $T^{*}$ are size dependent.

We did the same analysis for all the values of $\sigma$ studied in this mean field region and found that the Tsallis collapse works very well for temperatures, as in the case of $\sigma=0.30$, not too near $T_{c}$. In Table I we present the values of $a_{0}$ for each value of $\sigma$ studied.

Summarizing, our scaling proposal (the generalized Tsallis scaling) works very well in the mean-field region. That means the constant $a_{0}$ is well defined and the collapse obtained covers a wide range of temperature. In this way, considering temperatures not too near the critical one, we have demonstrated that this proposal allows us to connect the behavior of a finite system with different sizes and the bulk behavior.

\section{B. Long-range region}

On the one hand, we analyzed the critical finite-size scaling for the case of $\sigma>0.5$. As in the mean-field region, the relevant observables show power-law behaviors.

Figure 7 shows the same log-log plots that Fig. 3 but obtained for the interaction $\sigma=0.75$. As we can see, the data clearly suggest a power-law behavior ranging from $N=50$ to $N=5000$. In this figure the solid lines are power-law fits of Eqs. (4)-(6). The values of the exponents obtained for $\sigma=0.75$ and $\sigma=0.90$ are given in Table II, where we include
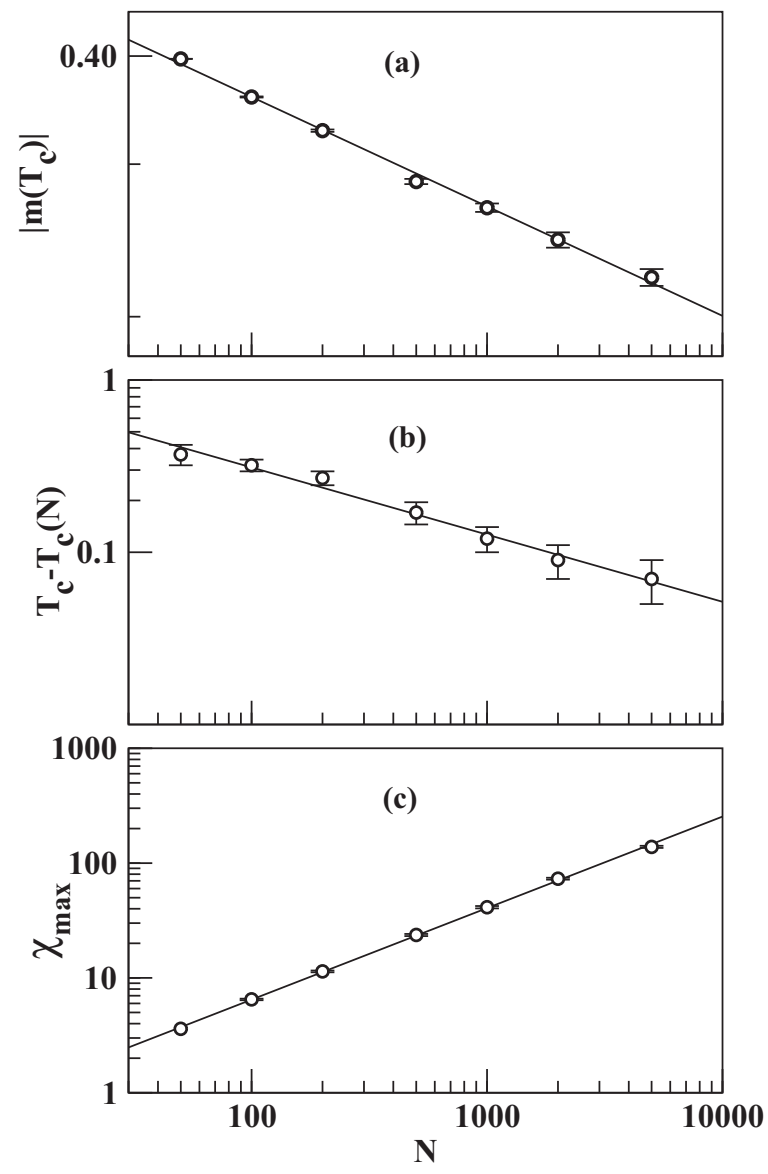

FIG. 7. The same as Fig. 3 for the case of $\sigma=0.75$. The fitted exponents are listed in Table II.

the known values from the literature for comparison. We can observe that the exponents fitted by finite-size scaling are close to those obtained from the RG theory [47] and from simulations using a short-time dynamic technique [27,39]. However, the exponents $v$ and $\gamma$ are still in clear disagreement with these accepted values. Again, we attribute these discrepancies to the small sizes of our simulated systems. For larger systems, we expect the values of the exponents approach the known values from the literature.

Figure 8 shows the collapse obtained for $|m|$ and $\chi_{T}$ for the case of $\sigma=0.75$, analogous to the one shown in Fig. 4, using our estimated exponents listed in Table II. In the case of Fig. 8(a) the collapse for the absolute magnetization works correctly in the range $-1.5 \lesssim \psi \lesssim 1$, while in Fig. 8(b) it works in the range $0 \lesssim \psi \lesssim 1.5$. These narrow ranges are similar to the mean-field behavior shown in Fig. 4. In the case of $\sigma=0.90$ (not shown) a similar behavior is observed.

On the other hand, our generalized Tsallis conjecture can still be applied in the long-range region $(0.5<\sigma<1)$. It is worth mentioning that Eq. (17) predicts that an increment in the value of $\sigma$ strongly reduces the difference between data corresponding to different sizes. In fact, we observed an enormous difference for $\sigma=0.1$, while for $\sigma=0.5$, the finitesize effects were smaller. In this way, from the generalized Tsallis conjecture, due to the LR interaction we expect weaker FSEs than in the mean-field region. 
TABLE II. Summary of the results obtained in the LR region using different range interactions. The critical exponents $\beta, 1 / \nu$, and $\gamma$ taken from the cited references are listed along those obtained in this work by means of finite-size scaling [Eqs. (4)-(6)], which are shown in the columns indicated by $\left({ }^{*}\right)$. In the second column we list the critical temperature used in the fits of Eqs. (4) and (5). The parameter $a_{0}$, adjusted to generalize the Tsallis conjecture, is shown in the last column.

\begin{tabular}{|c|c|c|c|c|c|c|c|c|}
\hline$\sigma$ & $T_{c}$ & $\beta$ & $\left(^{*}\right)$ & $1 / v$ & $\left(^{*}\right)$ & $\gamma$ & $(*)$ & $a_{0}$ \\
\hline \multirow[t]{2}{*}{0.75} & 2.669 & $0.227(15)[27]$ & $0.32(5)$ & $0.48(2)[27]$ & $0.39(6)$ & $1.54(6)[27]$ & $2.0(3)$ & 20 \\
\hline & & $0.262[47]$ & & $0.4765[47]$ & & $1.574[47]$ & & \\
\hline 0.90 & 2.00 & $0.119(15)[39]$ & $0.13(4)$ & $0.437(36)[39]$ & $0.30(5)$ & $2.05(19)[39]$ & $3.1(8)$ & 48 \\
\hline
\end{tabular}

Figures 9(a) and 10(a) show the absolute magnetization for $\sigma=0.75$ and $\sigma=0.90$, respectively. In the ferromagnetic phase, FSEs are present for $0.60 \lesssim T / T_{c}<1$ for both values of $\sigma$, which is a shorter range than the one observed in the mean-field region (see Fig. 3). In the same way as in Fig. 5(a), Figs. 9(b) and 10(b) show the transformation of the temperature, given by the generalized Tsallis conjecture, applied to the data of Figs. 9(a) and 10(a), respectively. The adjusted values of the effective-range constant are $a_{0}=20$ and $a_{0}=48$ as listed in Table II. Closer to $T_{c}$, our results, from the infinite-image method, show size dependence for the system sizes employed in this work, and so we have no data of the bulk in the range $0.96 \lesssim T / T_{c} \lesssim 1.05$ (the black dashed lines in Figs. 9 and 10 are only schematic). We note that for $N \geqslant 1000$ the generalized Tsallis conjecture produces,
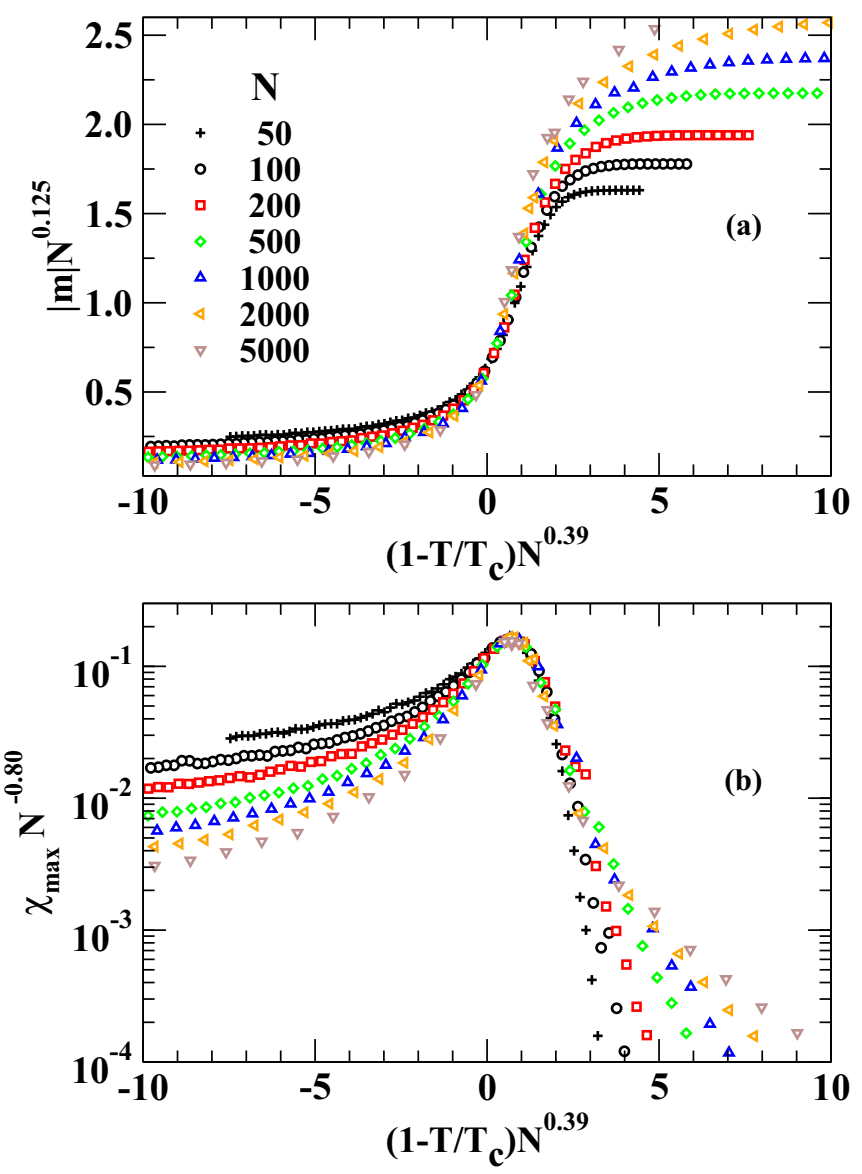

FIG. 8. (a, b) The collapses for magnetization and susceptibility, respectively, using the exponents calculated in this work, for the case $\sigma=0.75$. as in the case of the mean-field region, a good collapse in the region $T / T_{c} \lesssim 0.96$. Data corresponding to smaller systems $(100 \leqslant N \leqslant 500)$ are clearly away from the collapse with the bulk. However, we found that by taking a more general form for the effective distance $R_{0}$, i.e., by adding an additional constant term $R_{0}=c t e+N / a_{0}$, the smaller lattices also can be included in the collapse. The insets of Figs. 9(b) and 10(b) show the collapse obtained using the effective distances $R_{0}=7.0+N / 20$ and $R_{0}=6.0+N / 48$, respectively. As is observed in these insets, only curves coming from the smaller sizes are sensitive to the incorporation of the constant term in $R_{0}$. Therefore, this new term is determined exclusively by the data coming from the smaller sizes, and $a_{0}$ remains unchanged.
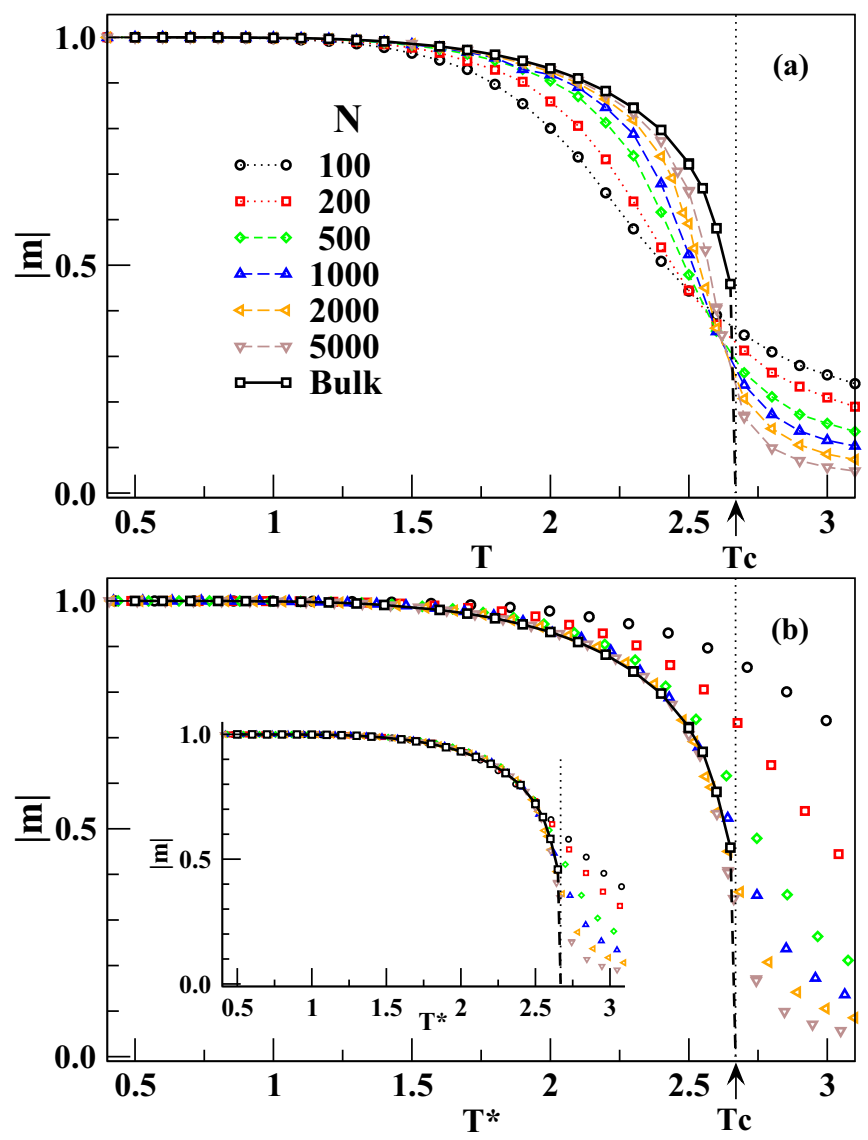

FIG. 9. (a) Absolute magnetization against the temperature $T$ for $\sigma=0.75$. (b) The same data against the scaled temperature $T^{*}$ calculated using the generalized Tsallis conjecture with $a_{0}=20$. The arrows and the vertical dotted line indicate the critical temperature $T_{c}$ (see Table II). The insets of Fig. (b) show the collapse obtained using the effective distances $R_{0}=7.0+N / 20$. 


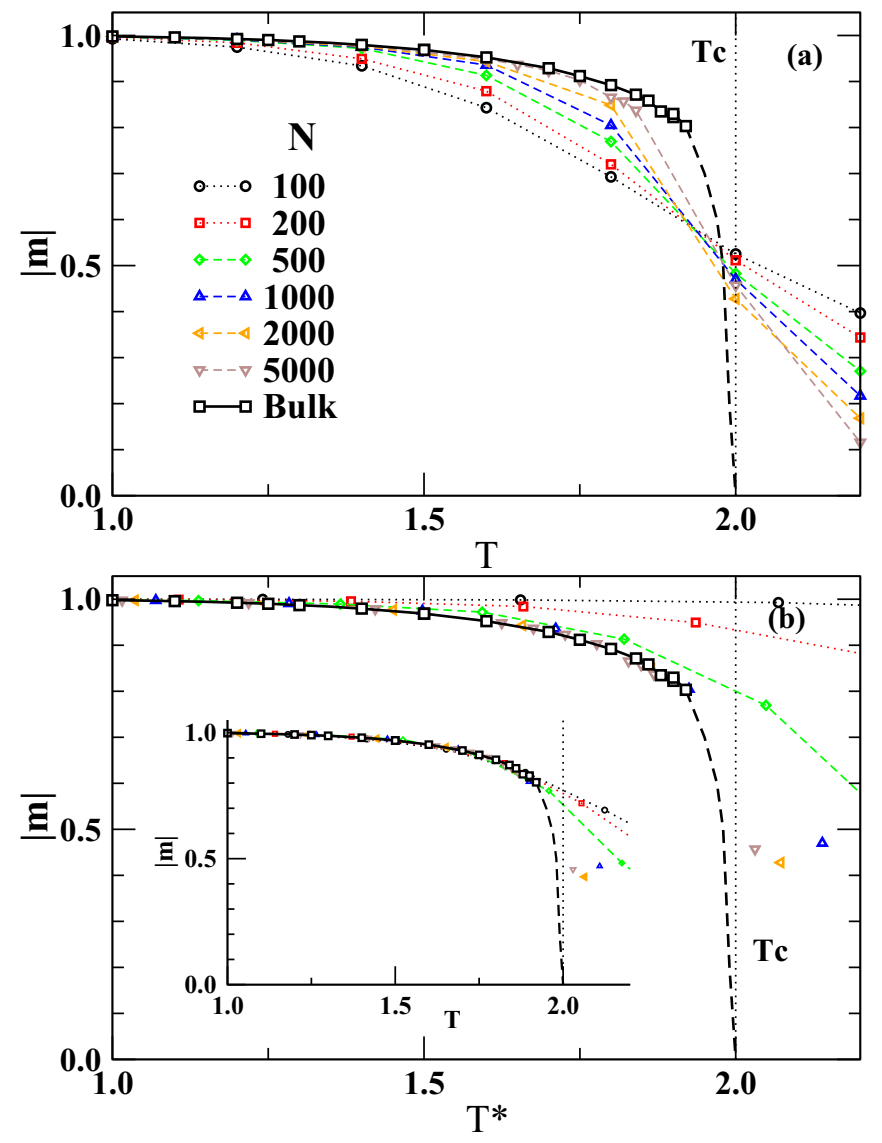

FIG. 10. Same as Fig. 9 for $\sigma=0.90$ with $a_{0}=48$. The inset of panel (b) shows the collapse obtained using the effective distances $R_{0}=6.0+N / 48$.

Summarizing: our scaling proposal works very well in the long-range region for not too small sizes. As in the case of the mean-field region, $a_{0}$ is well defined, and the collapse obtained covers a wide range of temperature. For very small systems $(100 \leqslant N \leqslant 500)$ the simplest modification in the definition of $R_{0}$ makes the quality of the proposed scaling comparable to that obtained in the mean-field region, across all the sizes and temperatures studied in this work.

We remark that in both cases, mean-field and long-range regions, the scaled temperature $T^{*}(\sigma, N)$, given by our scaling proposal (17), is determined completely by the adjusted constant $a_{0}$. We interpreted the constant $a_{0}$ as a factor giving, for a system of size $N$, an effective range $R_{0}=N / a_{0} . R_{0}$ is a measure of the average distance until which the interaction is relevant. The value of $a_{0}$ listed in Tables I and II shows an increment with the interaction parameter $\sigma$, in particular in the crossover from mean-field to long-range regions. For the mean-field region $a_{0}$ is typically small $\left(a_{0} \leqslant 5\right)$, which means the effective range is similar to the case of systems with PBCs $\left(R_{0}=N / 2\right)[22,43]$. In contrast, for the long-range region, the parameter $a_{0}$ is bigger than in the mean-field region, $20 \leqslant a_{0}$, i.e., one order of magnitude more. This increment, with increasing $\sigma$, makes the differences between systems with $\mathrm{OBCs}$ and systems with $\mathrm{PBC}$ more noticeable in the LR region than in the mean-field one.

\section{CONCLUSIONS}

We studied the finite-size effects in finite systems dominated by long-range interactions. We analyzed two different approaches to treat these effects and used a one-dimensional simple model with open boundary conditions, where the decay of long-range interactions is controlled by the parameter $\sigma$.

On the one hand, relevant observables show the power-law behavior expected from the finite-size scaling theory. In the mean-field region $(0<\sigma \leq 0.5)$ we have found, even for the small systems studied in this work, that the fitted exponents are mostly in agreement with the finite-size scaling theory presented recently for open boundary conditions [34]. Only the length correlation critical exponent, for the interaction $\sigma=$ 0.5 , shows an effective exponent significantly different from the predicted one. In the long-range region $(0.5<\sigma<1)$, near the critical point, a good collapse of data from systems with different sizes is observed supporting the proposed ansatz of finite-size scaling theory. However, the values fitted for the critical exponents are slightly different from the accepted ones in the literature. This difference shows that the application of this theory requires a more careful analysis, which is beyond of the focus of this work. Finally, in both regions, the collapses obtained have a narrow interval of validity in the scaling variable $\psi=\left|1-T / T_{c}\right| N^{1 / v}$, i.e., $-2 \lesssim \psi \lesssim 2$, or narrower.

On the other hand, we have shown that our proposal of a generalized Tsallis conjecture is successful to describe a wide interval of temperatures out of $T_{c}$ in both the mean-field and long-range regions. It consists of proposing an effective strength of interaction given by $R_{0}=N / a_{0}$. By adjusting the constant $a_{0}$, the only free parameter in this approach, it is possible to compare the results corresponding to different sizes using a scaled temperature $T^{*}(\sigma, N)$. Basically, different sizes produce the same results if they are at the same scaled temperature $T^{*}$. The physical meaning of $T^{*}$ is the temperature at which the bulk should be to have a similar behavior. In the long-range region, very small sizes deviate from this scaling, but we found that, by adding a constant term in $R_{0}$, small systems also can be included in the above comparison. Also, according to our interpretation, we found that this effective range decreases when the interaction decreases (i.e., when $\sigma$ increases). In conclusion, we show that the generalized Tsallis conjecture can be used empirically to predict the behavior of a finite system based on the bulk behavior and also to relate the behavior of systems with different sizes.

Unlike the finite-size scaling theory, where several parameters are needed (critical temperature and exponents), the generalized Tsallis conjecture uses only one empirical parameter. This generalized Tsallis scaling is somehow complementary to the finite-size scaling theory because its range of validity encompasses regions far from the critical one.

Due to the above reasons, we expect that this results will contribute to interpreting and predicting the behavior of real small systems in many experiments.

\section{ACKNOWLEDGMENTS}

This work was financially supported by CONICET, UNLP, and ANPCyT (Argentina). We thank UnCaFiQT (SNCAD) for computational resources. 
[1] Magnetic Properties of Fine Particles, edited by J. L. Dorman and D. Fiorani (North-Holland, Amsterdam, 1992).

[2] M. Kishimoto, Y. Sakurai, and T. Ajima, J. Appl. Phys. 76, 7506 (1994).

[3] R. F. L. Evans, W. J. Fan, P. Chureemart, T. A. Ostler, M. O. A. Ellis, and R. W. Chantrell, J. Phys.: Condens. Matter 26, 103202 (2014).

[4] C.-b. Rong, D. Li, V. Nandwana, N. Poudyal, Y. Ding, Z. L. Wang, H. Zeng, and J. P. Liu, Adv. Mater. 18, 2984 (2006).

[5] O. Hovorka, S. Devos, Q. Coopman, W. J. Fan, C. J. Aas, R. F. L. Evans, X. Chen, G. Ju, and R. W. Chantrell, Appl. Phys. Lett. 101, 052406 (2012).

[6] M. O. A. Ellis and R. W. Chantrell, Appl. Phys. Lett. 106, 162407 (2015).

[7] A. Lyberatos, D. Weller, G. J. Parker, and B. C. Stipe, J. Appl. Phys. 112, 113915 (2012).

[8] A. Lyberatos, D. Weller, and G. J. Parker, J. Appl. Phys. 114, 233904 (2013).

[9] R. H. French et al., Rev. Mod. Phys. 82, 1887 (2010).

[10] N. Goldenfeld, Lectures on Phase Transitions and the Renormalization Group (Addison-Wesley, New York, 1992).

[11] V. Privman (ed.), Finite-Size Scaling and Numerical Simulations of Statistical Systems (World Scientific, Singapore, 1990).

[12] K. Binder and D. Heermann, Monte Carlo Simulations in Statistical Physics: An Introduction, Fourth edition (Springer-Verlag, Berlin, Heidelberg, New York, 2002)

[13] J. G. Brankov, D. M. Dantchev, and N. S. Tonchev, The Theory of Critical Phenomena in Finite-Size Systems-Scaling and Quantum Effects (World Scientific, Singapore, 2000).

[14] D. M. Dantchev, H. W. Diehl, and D. Gruneberg, Phys. Rev. E 73, 016131 (2006).

[15] D. M. Dantchev, F. Schlesener, and S. Dietrich, Phys. Rev. E 76, 011121 (2007).

[16] G. Valchev and D. M. Dantchev, Phys. Rev. E 96, 022107 (2017).

[17] H. Chamati and N. S. Tonchev, Phys. Rev. E 63, 026103 (2001).

[18] H. Chamati and N. S. Tonchev, Mod. Phys. Lett. B 17, 1187 (2003).

[19] J. Wang, W. Wu, F. Zhao, and G.-m. Zhao, Appl. Phys. Lett. 98, 083107 (2011)

[20] X. Batlle and A. Labarta, J. Phys. D: Appl. Phys. 35, R15 (2002).

[21] C. Tsallis, Fractals 3, 541 (1995).
[22] P. Jund, S. G. Kim, and C. Tsallis, Phys. Rev. B 52, 50 (1995).

[23] R. F. S. Andrade and S. T. R. Pinho, Phys. Rev. E 71, 026126 (2005).

[24] D. Ruelle, Commun. Math. Phys. 9, 267 (1968).

[25] F. J. Dyson, Commun. Math. Phys. 12, 91 (1969).

[26] M. Ainzenman and R. Fernandez, Lett. Math. Phys. 16, 39 (1988).

[27] D. E. Rodriguez, M. A. Bab, and E. V. Albano, J. Stat. Mech. (2011) P090007.

[28] S. A. Cannas, C. M. Lapilli, and D. A. Stariolo, Int. J. Mod. Phys. C 15, 115 (2004).

[29] S. A. Cannas, Phys. Rev. B 52, 3034 (1995).

[30] S. A. Cannas and F. A. Tamarit, Phys. Rev. B 54, R12661 (1996).

[31] E. Luijten and H. W. J. Blöte, Phys. Rev. B 56, 8945 (1997).

[32] D. P. Landau, Phys. Rev. B 13, 2997 (1976).

[33] E. Flores-Sola, B. Berche, R. Kenna, and M. Weigel, Eur. Phys. J. B 88, 28 (2015).

[34] E. Flores-Sola, B. Berche, R. Kenna, and M. Weigel, Phys. Rev. Lett. 116, 115701 (2016).

[35] R. Kenna and B. Berche, Scaling and finite-size scaling above the upper critical dimension, in Order, Disorder, and Criticality: Advanced Problems of Phase Transition Theory, Vol. 4, edited by Yu. Holovatch (World Scientific, Singapore, 2015).

[36] M. E. Fisher, S. K. Ma, and B. G. Nickel, Phys. Rev. Lett. 29, 917 (1972).

[37] Z. Glumac and K. Uzelac, J. Phys. A: Math. Gen. 21, L421 (1988).

[38] Z. Glumac and K. Uzelac, J. Phys. A: Math. Gen. 22, 4439 (1989).

[39] Y. Tomita, J. Phys. Soc. Japan 78, 014002 (2009).

[40] E. Bayong, H. T. Diep, and T. T. Truong, J. Appl. Phys. 85, 6088 (1999).

[41] E. Bayong and H. T. Diep, Phys. Rev. B 59, 11919 (1999).

[42] C. Tsallis, Braz. J. Phys. 29, 1 (1999).

[43] L. A. del Pino, P. Troncoso, and S. Curilef, Phys. Rev. B 76, 172402 (2007).

[44] S. Curilef, Int. J. Mod. Phys. C 11, 629 (2000).

[45] S. Curilef, L. A. del Pino, and P. Orellana, Phys. Rev. B 72, 224410 (2005).

[46] R. Rüger and R. Valentí, Phys. Rev. B 86, 024431 (2012).

[47] K. Binder and E. Luijten, Phys. Rep. 344, 179 (2001). 